\title{
Electro-ultrafiltration study on Aldrich humic substances with different molecular weights
}

\author{
Hsin-Hua Lee ${ }^{\mathrm{a}}$, Yu-Hsiang Weng ${ }^{\mathrm{b}}$, Kung-Cheh $\mathrm{Li}^{\mathrm{c}, *}$ \\ a Taiwan Semiconductor Manufacturing Company Limited, No. 25, Li-Hsin Road, Hsinchu Science Park, HsinChu 300, Taiwan, ROC \\ ${ }^{\mathrm{b}}$ Institute of Nuclear Energy Research, Atomic Energy Council, Executive Yuan, 1000 Wenhua Road, Chiaan Village, Lungtan, Taoyuan 325, Taiwan, ROC \\ ' Graduate Institute of Environmental Engineering, National Taiwan University, 71 Chou-Shan Road, Taipei 106, Taiwan, ROC
}

\section{A R T I C L E I N F O}

\section{Article history:}

Received 22 June 2007

Received in revised form 5 March 2008

Accepted 12 March 2008

\section{Keywords:}

Molecular weight (MW)

Electro-ultrafiltration (EUF)

Aldrich humic substances (AHSs)

Gel fractionation chromatography (GFC)

Atomic force microscopy (AFM)

\begin{abstract}
A B S T R A C T
This study examined the effect of molecular weight (MW) fractions on electro-ultrafiltration (EUF). MW fractions fractionated from Aldrich humic substances (AHSs) were defined as G1 (3000 to >20,000 Da), G2 ( 500 to $3000 \mathrm{Da}$ ) and G3 ( $<400$ to $500 \mathrm{Da}$ ) by using gel fractionation chromatography (GFC). G3 exhibited the largest flux decline in the absence of an electric field. The decline may be attributed to the relatively small MW and apparent electrophoretic mobility (AEM) of G3. The result from atomic force microscopy (AFM) roughness analysis suggested that G3 possibly blocked the membrane pore, but G1 and G2 were possibly accumulated on the membrane surface. In the presence of an electric field, both flux and removal were simultaneously increased due to the electrokinetic phenomena. However, the extent of the flux decline was higher for G1 than that for G3. It was postulated that electrochemical reactions changed the characteristics of membrane and AHSs.
\end{abstract}

(C) 2008 Elsevier B.V. All rights reserved.

\section{Introduction}

Membranes with large pore sizes $(>0.01 \mu \mathrm{m})$, such as microfiltration (MF) and ultrafiltration (UF), have received great attention due to their enhanced performance to remove pathogens, as compared with traditional water treatment processes [1]. In addition, the replacement or cooperation of traditional water treatment processes with an advanced technology, such as membrane filtration, provides an alternative means to meet stringer environmental regulations. However, MF and UF suffer from fouling, which would deteriorate permeation flux, and therefore operation cost is increased. Many studies showed that humic substances (HSs) are one of the most significant fouling agents $[2,3]$.

Although membrane fouling by HSs has been studied extensively [3-7], the results regarding which molecular weight (MW) fraction of HSs is the major fouling component are contradictory. For example, Lin et al. [4] examined the effect of MW on UF with and without powder active carbon (PAC) pretreatment. Without PAC treatment, flux decline was more pronounced for the large MW fraction than that for the small MW fraction. In addition, the fraction that could not be adsorbed by PAC also caused significant flux decline. Yuan and Zydney [8] also reported that large MW fraction induced severe fouling. However, several studies showed that

\footnotetext{
* Corresponding author. Tel.: +886 2 23698112; fax: +886223638173

E-mail address: kcli@ntu.edu.tw (K.-C. Li).
}

small MW fraction with hydrophilic characteristic has significant influence on flux decline $[3,6,7]$.

In order to counter fouling, an electric field is applied during UF. This is called "electrofiltration" in which the movement of foulant, such as HSs, was influenced by the applied electric field and its apparent electrophoretic mobility (AEM). Since HSs are negatively charged in natural water system with neutral $\mathrm{pH}$, the anode is placed on the feed side and cathode is placed on filtrate side to attract HSs and to prevent them from accumulating on membrane surfaces or pores. It is noted that polarity should be reversed if the foulant is positively charged [9]. Generally, the AEM of HSs ranges from -3.0 to $-5.0 \times 10^{-8} \mathrm{~m}^{2} \mathrm{~V}^{-1} \mathrm{~s}^{-1}$ depending on sources and solution environments $[10,11]$. In a study on capillary electrophoresis of a peat HSs, De Nobili et al. [10] reported a similar fingerprint for different MW HSs. While efficiency of electrofiltration is primarily based on AEM of species in solution, it was expected that fouling could be controlled during electroultrafiltration (EUF).

Although much has been reported to eliminate fouling and increase removal of HSs by electrofiltration [12-15], there are still gaps in the literature about the effect of HSs MW fractions on the performance of EUF. In this study, gel fractionation chromatography (GFC) was used to separate HSs into different MW groups. Flux and HSs removals were determined for each EUF experiment. Furthermore, membrane morphology was measured by atomic force microscopy (AFM) to understand the change of membrane surface before and after fouling. 


\section{Materials and methods}

\subsection{Preparation of AHSs solution}

The synthetic raw water was prepared by filtering $5 \mathrm{~g} / \mathrm{L}$ humic acid (sodium salt, purchased from Aldrich Chemical Co.) solution through a $0.45 \mu \mathrm{m}$ filter, giving a stock solution with a dissolved organic carbon (DOC) concentration of approximately $250 \mathrm{mg} / \mathrm{L}$. The stock solution of Aldrich humic substances (AHSs) was stored in refrigerator at $4{ }^{\circ} \mathrm{C}$ before use. Sodium chlorine was used as background electrolyte. The DOC concentration was adjusted to $5 \mathrm{mg} / \mathrm{L}$ with a conductivity of $50 \mu \mathrm{S} / \mathrm{cm}$ and $\mathrm{pH} 7$ for each EUF experiment.

\subsection{Fractionation of AHSs by molecular weight}

The AHSs stock solution was fractionated by gel fractionation chromatography. The gel used in this study was Sephadex G-75 type which is suitable for preparing the dextran and global protein in the range from 1000 to $80,000 \mathrm{Da}$. This method has been used previously to fractionate AHSs $[2,4]$. Polyethylene glycols (PEGs) with MW of 20,000, 12,000,6000, 1500 and 400 Da (Merck) were used as standards for calibration. It is well known that solution environments have significant impacts on MW fractionation of HSs. In this study, distilled water was used as eluent. No buffers and $\mathrm{pH}$ adjustments were applied during fractionation experiments. The elution at different time intervals was collected by an automatic fraction collector. Samples at each tube were analyzed by using UV spectroscopy and DOC analyzer to obtain a MW distribution curve. In principle, large MW molecules are excluded earlier than smaller ones, because they are unable to travel through the gel pores. High correlation between the exclusion time and the MW was obtained. According to the MW distribution curve calibrated with PEGs, AHSs samples were divided into three MW groups, denoted as G1 (3000 to >20,000 Da), G2 (500 to $3000 \mathrm{Da}$ ) and G3 ( $<400$ to $500 \mathrm{Da}$ ). The recovery of the AHSs in this study was approximately $73 \%$. Table 1 shows typical characteristics of fractionated AHSs.

\subsection{Filtration modules and experiment protocol}

A laboratory scale EUF module was constructed by assembling two plates with one silicon plate in between. The electrodes which were located on two sides of the membrane were consisted of platinum and titanium. The distance between two electrodes was $4 \mathrm{~mm}$, and the filtration area was $16.75 \mathrm{~cm}^{2}$. The detailed layout of the cell was shown previously [14].

The membranes used were $100 \mathrm{kDa}$ polyacrylonitrile (PAN) UF membranes (purchased from Osmosis). This membrane was hydrophilic with a contact angle of $4^{\circ}$. Before the experiment was conducted, the membrane was soaked in Milli-Q water and then filtered with Milli-Q water for $1 \mathrm{~h}$ to remove preservatives and stabilize the membrane. Thereafter, AHSs solutions with different MW distributions were pumped into the cell. The transmembrane pressure was $49-98 \mathrm{kPa}$ and crossflow velocity was $0.12 \mathrm{~m} / \mathrm{s}$. It should be noted that all the filtrate and the concentrate were recycled back to the feed tank except those were taken for chemical analyses. Experiments were conducted at room temperature $\left(\sim 20^{\circ} \mathrm{C}\right)$.
Flux reduction $\left(J_{\text {red }}\right)$ is defined in Eq. (1), where $J_{\mathrm{i}}$ is the flux at initial $(t=0)$ and $J_{\mathrm{f}}$ is the flux at final $(t=300 \mathrm{~min})$. A high $J_{\text {red }}$ means a higher potential to experience flux decline.

$J_{\text {red }}=\frac{J_{\mathrm{i}}-J_{\mathrm{f}}}{J_{\mathrm{i}}} \times 100 \%$

\subsection{Water quality analyses}

The total organic carbon (TOC) was analyzed with an Organic Carbon Analyzer (model OI Analytical 1010). Conductivity and $\mathrm{pH}$ were measured with potentiometers Suntex model SC-120 and Consort model C831. Additionally, AHSs were also quantified as $\mathrm{UV}_{254}$ measured with a Cintra $20 \mathrm{UV}$-Visible spectrometer at $\mathrm{pH}$ $7.0 \pm 0.1$. Electrophoretic mobility measurements were made by Malvern Zeta Sizer 3000, giving an AEM for each sample.

AHSs removal efficiency ( $R$ ) was calculated by Eq. (2), where $C_{0}$ and $C_{\mathrm{f}}$ are initial UV absorbance in feed and the final UV absorbance in filtrate, respectively.

$R=\frac{C_{0}-C_{\mathrm{f}}}{C_{0}} \times 100 \%$

The filtration resistance was calculated by Darcy's law in Eq. (3).

$R_{f}=\frac{\Delta P}{J \eta}$

In this equation, $R_{\mathrm{f}}$ is the filtration resistance $\left(\mathrm{m}^{-1}\right), \Delta P$ is transmembrane pressure $(\mathrm{Pa}), J$ is flux $(\mathrm{m} / \mathrm{s})$ and $\mu$ is viscosity $\left(\mathrm{N} \mathrm{s} \mathrm{m}^{-2}\right)$.

\subsection{Membrane morphology and zeta potential analyses}

AFM was used to analyze membrane morphology. Measurements were made by Digital Instrument. The samples were dried at room temperature and a specimen was cut before AFM analysis. The scan size for all the specimens was $5 \mu \mathrm{m} \times 5 \mu \mathrm{m}$. The average roughness can be calculated from the computer software.

Membrane streaming potential was measured using a device consisting of two parallel-plates with two $\mathrm{IrO}_{2}$ electrodes at each side. The measurement was carried out by varying applied pressure corresponding to different currents. The tangential streaming potential can be calculated using Helmholtz-Smoluchowski equation [16].

\section{Results and discussion}

\subsection{Fractionation of AHSs by GFC}

Fig. 1 shows MW distribution of AHSs using a GFC technique and calibrated with PEGs. G1, G2 and G3 groups represented large(3000 to $>20,000 \mathrm{Da}$ ), medium- (500 to $3000 \mathrm{Da}$ ) and small- ( $<400$ to $500 \mathrm{Da}$ ) sized organics in AHSs. There is a peak at around $10,000 \mathrm{Da}$, which dominated large MW fraction. This result is consistent with previous studies with the same separation technique and model compound $[2,4]$.

The characteristics of unfractionated and fractionated AHSs are listed in Table 1. Major DOC comes from the large MW component, which represents $50 \%$ of organic in stock solution. In addition, all

Table 1

Characteristics of different MW AHSs at $\mathrm{pH} \sim 5.6$

\begin{tabular}{|c|c|c|c|c|}
\hline MW group & Unfractionated & $\mathrm{G} 1(3000>20,000 \mathrm{Da})$ & G2 (500 to $3000 \mathrm{Da})$ & G3 $(<400$ to $500 \mathrm{Da})$ \\
\hline Stock (DOC mg/L) & 256 & 48 & 24 & 29 \\
\hline $\operatorname{AEM}\left(x 10^{-8} \mathrm{~m}^{2} \mathrm{~V}^{-1} \mathrm{~s}^{-1}\right)$ & -3.09 & -3.25 & -2.71 & -2.17 \\
\hline Zeta potential $(\mathrm{mV})$ & -39.3 & -41.4 & -34.4 & -27.7 \\
\hline
\end{tabular}




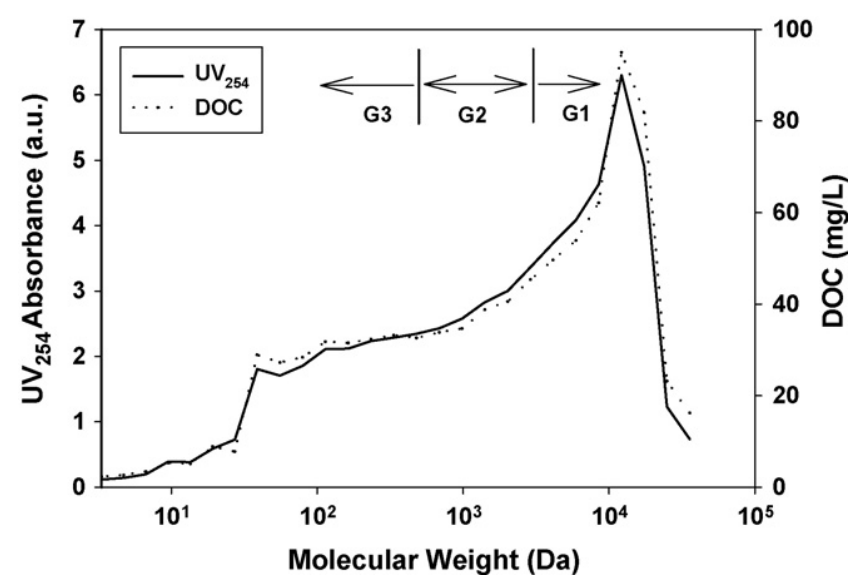

Fig. 1. Molecular weight distribution of AHSs.

of these three fractions were negatively charged from AEM measurements. AEM was higher for G1 than that for medium and small MW groups, i.e., G2 and G3. It was therefore postulated that the electric field would be less effective for G3 group than that for G1 and G2 groups. These AEM values were on the same order with our previous study [12] and measurements by capillary electrophoresis $[10,11]$. In addition, the AEM result suggested that AHSs charge is not independent of MW, although De Nobili et al. [10] reported a similar AEM for different MW fractions of HSs extracted from peat. This may be due to the differences in origin and measurement techniques employed.

It is well known that zeta potential of a spherical particle can be derived from electropohretic mobility according to Henry's equation [16] where $\mu$ is the electrophoretic mobility $\left(\mathrm{m}^{2} \mathrm{~V}^{-1} \mathrm{~s}^{-1}\right), \varepsilon$ is the dielectric constant of the solution $\left(\mathrm{C}^{2} \mathrm{~N}^{-1} \mathrm{~m}^{-2}\right), \varsigma$ is the zeta potential $(\mathrm{V}), \eta$ is the viscosity of solution $\left(\mathrm{N} \mathrm{s} \mathrm{m}^{-2}\right)$.

$\mu=\frac{2 \varepsilon \varsigma}{3 \eta} f(\alpha)$

While Henry's equation implicitly assumes that the zeta potential of a particle is low and neglect retardation effect, it is reasonably true in most environmental application. Both AEM and zeta potential of AHSs were reported here, although the real conformation and shape of AHSs are unknown. It is noted that zeta potentials were calculated in Helmholtz-Smoluchowski's limit. It is clear that zeta potential is proportional to electrophoretic mobility from Henry's equation.

\subsection{Effect of transmembrane pressure on flux}

Fig. 2 shows the effect of transmembrane pressure on flux. The Milli-Q water fluxes for clean membrane were 8.1, 6.2 and $4.2 \times 10^{-5} \mathrm{~m} / \mathrm{s}$ at 98,74 and $49 \mathrm{kPa}$, respectively. Therefore, the filtration resistance for a clean membrane was $1.25 \times 10^{12} \mathrm{~m}^{-1}$,as calculated by the Darcy's law in Eq. (3). The initial filtration flux of AHSs solution was slightly smaller than that of Milli-Q water. In comparison with its Mill-Q water flow, the flux reduction after $5 \mathrm{~h}$ operation was $15 \%, 22 \%$ and $27 \%$ at 49,74 and $98 \mathrm{kPa}$, respectively.

The application of electric voltage at $50 \mathrm{~V}$ raised the initial flux dramatically at all operational pressures. Flux fluctuation became greater, after applying voltages and reached a relatively stable value at $200 \mathrm{~min}$. A Similar phenomenon was observed from electrofiltration of AHSs [13], wastewater [17] and colloidal solution [18]. The initial flux for EUF was not only greater than that for UF of AHSs without voltage, but also greater than that for UF of Mill-Q water. After applying an electric field, flux enhancements were 34\%, 33\%

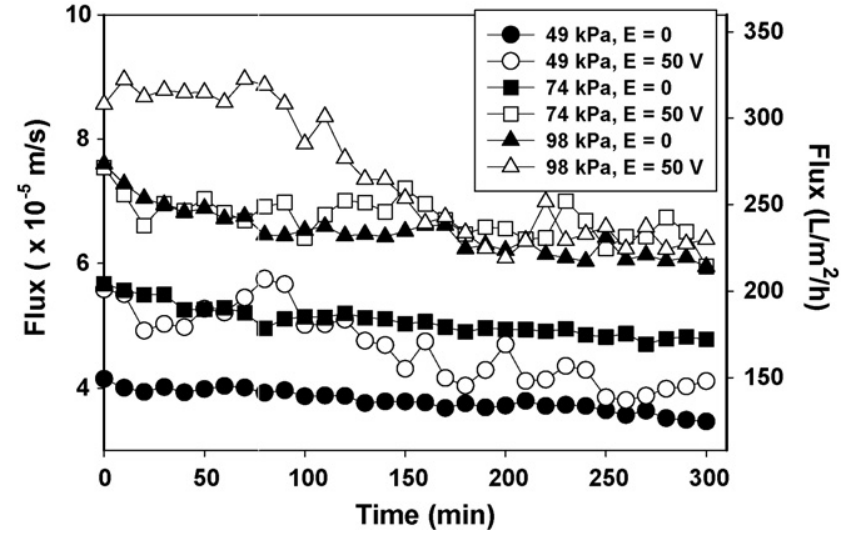

Fig. 2. Flux of AHSs at different pressures during EUF.

and $13 \%$ at 49,74 and $98 \mathrm{kPa}$, respectively. However, it could not avoid flux decline during EUF of AHSs. There were 25\%, 21\% 26\% flux reduction at 98,74 and $49 \mathrm{kPa}$, respectively, as compared to its initial value in the presence of electric field. Despite the flux decline occurred, the final flux in the presence of electric field remained higher than that in the absence of an electric field.

At beginning of the experiment, there were 26\%, 20\% and 6\% flux enhancement in comparison with its Mill-Q water flux at 49, 74 and $98 \mathrm{kPa}$, respectively. Since electrophoresis denotes the movement of species in the electric field, it was not able to account for the incremental flux in such a circumstance. Therefore, it is clearly that the incremental flux was the contribution of electroosmosis although electrophoresis did take place at the same time. Similar results were obtained by Weng and $\mathrm{Li}$ [12] with $100 \mathrm{kDa}$ polyvinylidene fluoride (PVDF) membranes and Weng et al. [14] with $100 \mathrm{kDa}$ PAN membranes during EUF of AHSs. They reported that electroosmotic flux during EUF of AHSs was in the range of 5-36\% which is in agreement with present study. In general, electroosmosis is dependent on the electric field, characteristics of membrane and solution properties. Interestingly, more than $100 \%$ enhancement was observed during electrofiltration of a polymer suspension at high voltage [19]. It is noted that the higher percentage of flux enhancement at low pressure condition was due to low permeate flux. The absolute values of the electroosmotic flow at different pressures were not significantly different.

\subsection{Effect of $M W$ fraction on flux}

In order to investigate more severe fouling scenarios, pressure was chosen at $98 \mathrm{kPa}$ during the experiments. Figs. 3(a) and 4 show that flux declined to $68 \%, 67 \%$ and $54 \%$ of its initial value for G1, G2 and $\mathrm{G} 3$ groups, respectively. This result suggested that $\mathrm{G} 3$ group, i.e., low MW organic, had higher potential to cause membrane fouling. Nevertheless, G1 and G2 groups also induced more than 30\% flux declination in this study. Other studies also indicated that small MW HSs had higher potential to cause flux decline than larger ones $[3,6,7]$. For instance, Howe and Clark [7] pointed out that low MW compound was the major contributor to fouling during filtration of natural water in US. They also showed that no fouling condition was found after removing the small MW foulants. In addition, the study by Carroll et al. [6] showed that the smallest MW component extracted from Moorabool River had a neutral-hydrophilic feature and caused the severest flux decline. Furthermore, Li and Chen [3] also reported that small MW fractions separated from AHSs tented to cause fouling in membrane pores.

However, our result was different from the studies by several researchers $[4,5]$. In the studies, it was shown that large MW frac- 

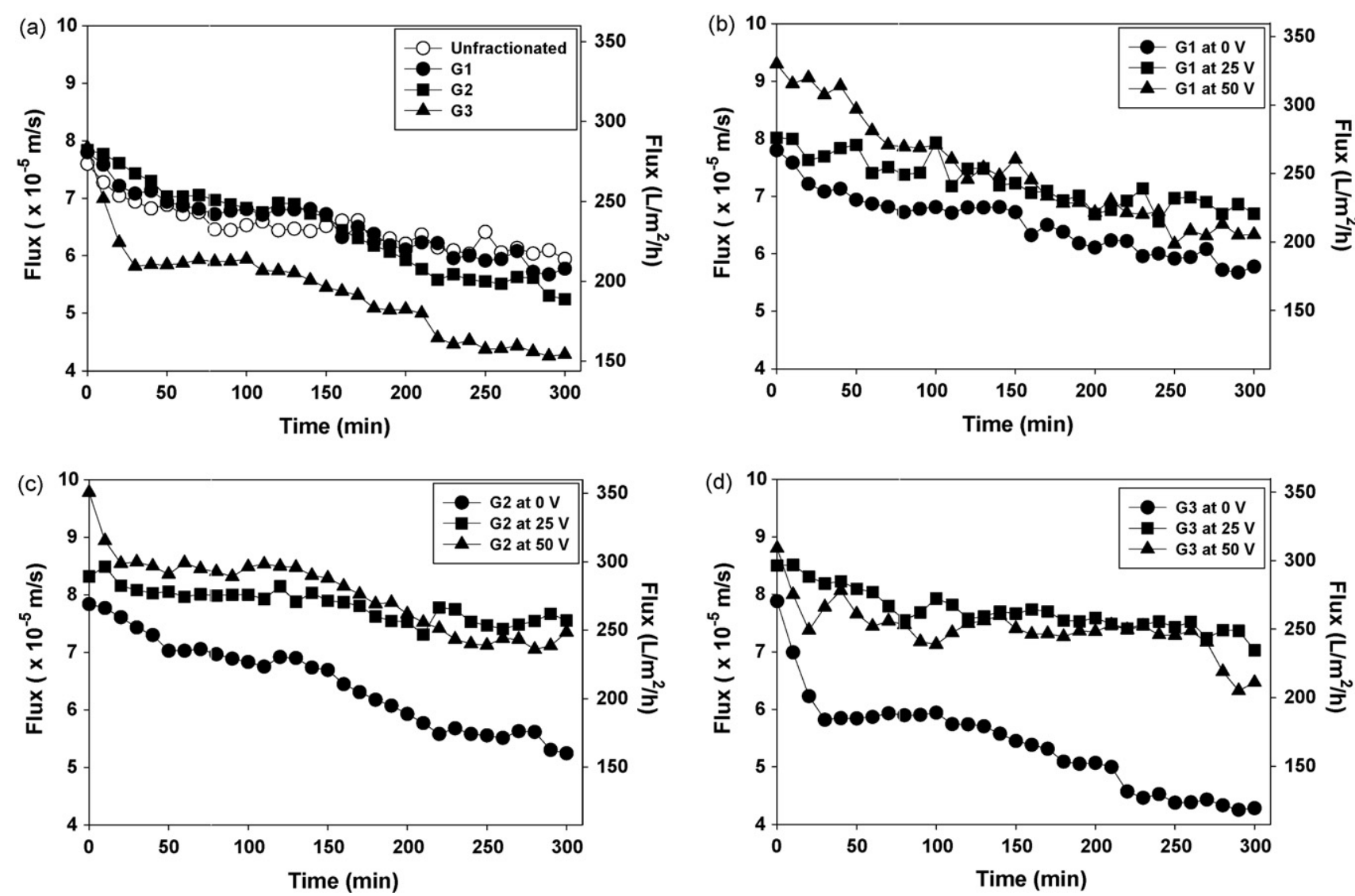

Fig. 3. Effects of different MW fractions of AHSs on EUF: (a) without voltage, (b) G1 fraction, (c) G2 fraction and (d) G3 fraction.

tion caused more severe fouling than small MW one. Yuan and Zydney [5] showed that HSs aggregates would cause fouling on the membrane surface and removing them can reduce fouling. However, their experiments were conducted in a dead-end mode, and the results may not be applicable to our study. The differences between this study and Lin's lie in different membrane materials [4]. The hydrophilic characteristics of PAN membrane might be preferential to adsorb G3 in membrane pore because of its lower charge and lower size (Table 1). Several researchers have pointed out the importance of membrane characteristics on fouling $[5,20,21]$. A different fouling tendency could be obtained when two UF membranes, i.e., one hydrophobic and one hydrophilic, were

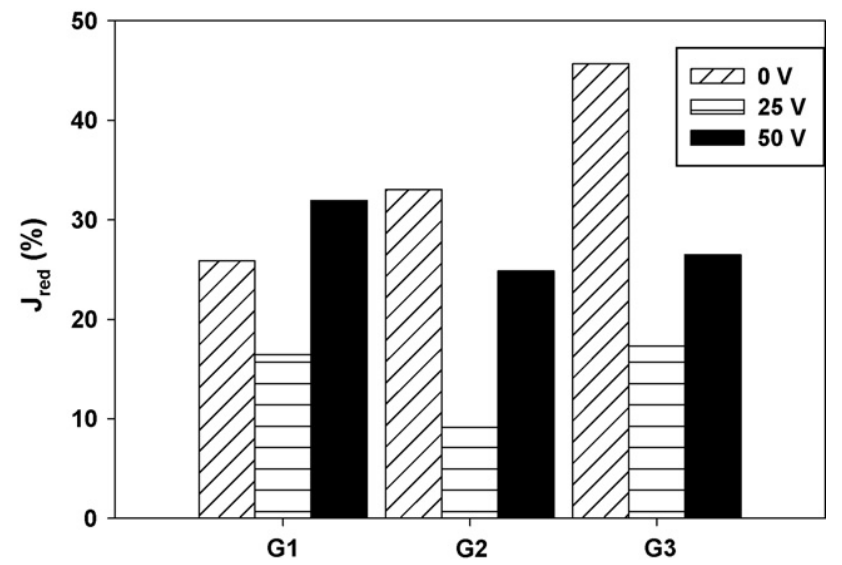

Fig. 4. Flux reductions during filtration of MW fractions at various electric voltages. used to treat water containing natural organic matter (NOM) and model compounds [20].

Fig. 5 shows the membrane images by AFM. AFM roughness analysis has been considered as an indicator to gain insight into membrane fouling because it is noninvasive and free of pretreatment $[20,21]$. Table 2 demonstrates that membrane roughness is not only dependent on membrane materials, but also on water qualities. Increased or decreased roughness after fouling was observed. Lee et al. [21] reported that particulate organic matter tended to deposit on the membrane surface and thereby increased membrane roughness after filtration. In contrast, AFM image of a clean UF membrane was not significantly different from that of membrane fouled by dissolved organic matter. It appeared that G3 group filled membrane pore and reduced roughness of membrane, whereas G1 and G2 groups were accumulated on the membrane surface and therefore increased membrane roughness. Thus, fouling induced by G1 and G2 might be retained on the membrane surface due to their larger MW.

Table 2 also demonstrates that streaming potential of the membrane during filtration of AHSs. It is clear that membrane streaming potential was less affected by filtering G3 group AHSs. Although AHSs and membrane were both negatively charged, it is inevitable to experience fouling during membrane filtration [5]. Since membrane is a pressure driven process, it is clear that both convective force towards membrane and the repulsive force coming from membrane charge should be considered. As observed from the experimental results, the convective force was greater than the repulsive force. This resulted in deposition of AHSs on the membrane surface or in the membrane pore. In addition, adsorption of AHSs on the membrane also decreased the membrane surface potential, which resulted in less repulsive force between the mem- 


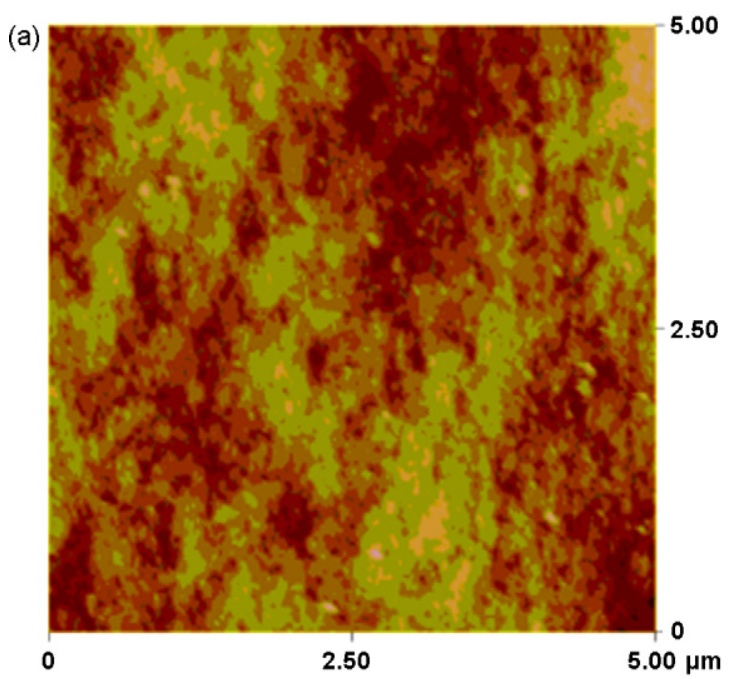

(b)

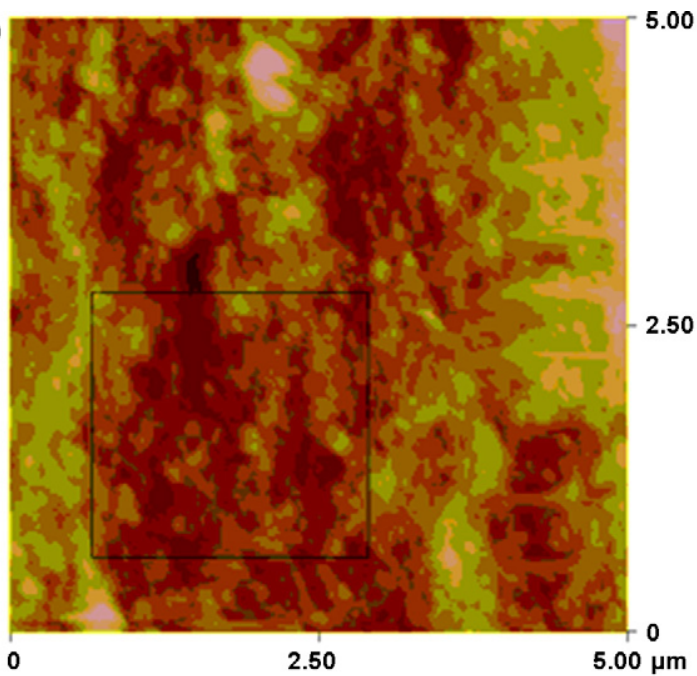

(c)

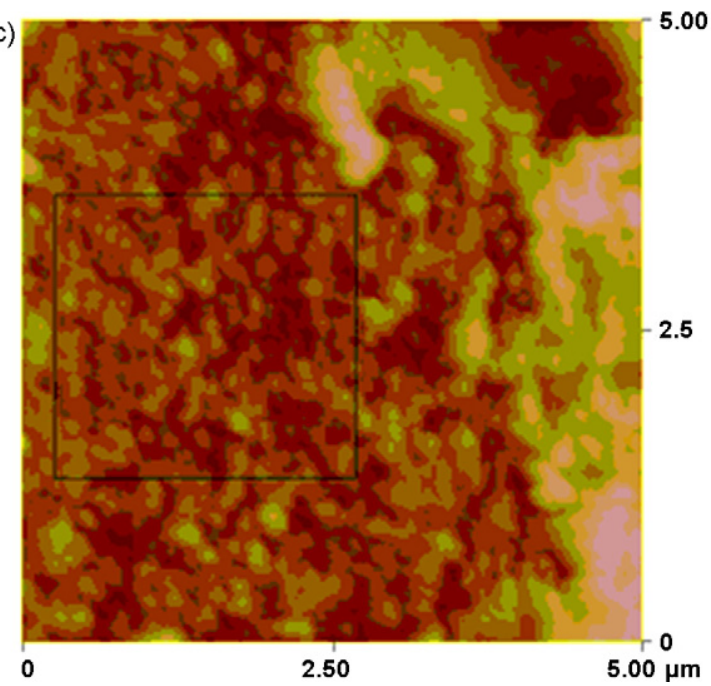

(d)

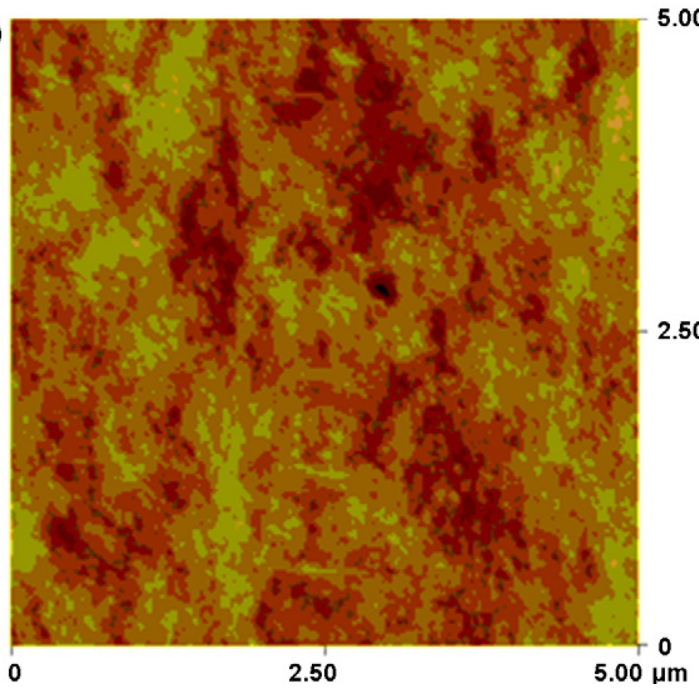

Fig. 5. AFM surface analysis on fouled membrane: (a) Mili-Q water, (b) G1 fraction, (c) G2 fraction and (d) G3 fraction.

brane and ASHs [5]. Thus, despite of larger zeta potential of G1 and G2 groups, flux decline occurred during filtration.

Fig. 3(b)-(d) shows the effect of MW on EUF. For all three fractions, flux increased after applying voltages. In general, flux increased as the applied electric field increased. Interestingly, flux at $50 \mathrm{~V}$ was higher than that at $25 \mathrm{~V}$ in the beginning, but a reverse trend was observed over time. Flux reduction analysis also showed a similar trend (See Fig. 4), i.e., $25 \mathrm{~V}$ performed better than $50 \mathrm{~V}$. A possible explanation for this could be the change in membrane surface property due to water hydrolysis near the electrodes. In general, $\mathrm{pH}$ would rise to 10 in cathode chamber and lower to 4 in anode chamber in our experimental conditions. According to the suggestions by the manufacturer, this membrane adapts to
$\mathrm{pH}$ ranging from 2 to 10 . Therefore, alkalinity solution at $\mathrm{pH} \sim 10$ was used to simulate the solution environment during EUF. The membrane was soaked in a $0.1 \mathrm{~N} \mathrm{NaOH}$ solution for $5 \mathrm{~h}$, and Mill-Q water filtration rate was measured before and after $\mathrm{NaOH}$ treatment. Fig. 6 shows that Mill-Q water flux decreased after $\mathrm{NaOH}$ pretreatment. It is postulated that $\mathrm{pH}$ changes due to water hydrolysis might affect the performance of the EUF. Although Yang et al. [17] have attributed the decrease in flux after elctromicrofilation to clogging of small particles into membranes, change of membrane property in terms of Mili-Q water flux was observed in this study. Recently, application of electrofiltration to remove nanoparticles from water was demonstrated by using inorganic membrane [22].

Table 2

Roughness and streaming potential of clean and fouled membranes

\begin{tabular}{|c|c|c|c|c|}
\hline Water matrix & 100 kDa PAN membrane $(\mathrm{nm})$ & 100 kDa PAN membrane ${ }^{a}(\mathrm{mV})$ & Water matrix & 100 kDa RC membrane ${ }^{b}(\mathrm{~nm})$ \\
\hline Clean & 3.6 & -11.55 & Clean & 22.9 \\
\hline G1 & 11.3 & -8.25 & Marne River & 26.8 \\
\hline G2 & 14.2 & -8.39 & Yffiniac River & 23.5 \\
\hline G3 & 3.0 & -9.32 & Brittany Reservoir & 20.2 \\
\hline & & & Silver Lake & 11.0 \\
\hline
\end{tabular}

\footnotetext{
a This study.

b Regenerated cellulose (RC) membrane, data from Lee et al. [21].
} 


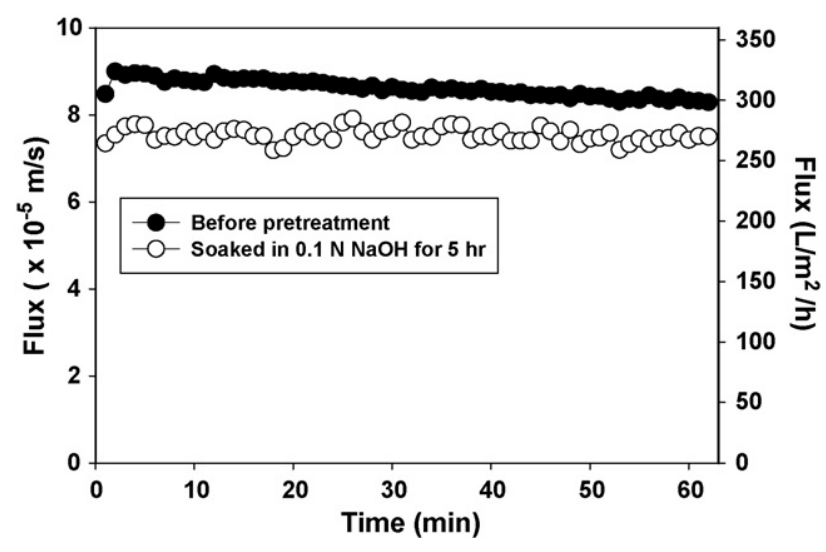

Fig. 6. Effect of membrane pretreatment on Mili-Q water flux.

Moreover, changes in AHSs characteristics might occur after EUF. Although the electrophoresis and electroosmosis are major effects during electrofiltration, electrochemical reactions do take place if the electrodes are not separated from the streams [14]. It has been shown that AHSs compositions changed but to a less extent after electrofiltration [13].

Fig. 7 summarizes the filtrate produced in various experimental conditions. Water produced in terms of volume was calculated by integration of flux versus time curve. In general, the higher the voltage, the more water produced. In average, the incremental volume was $20 \%$ and $24 \%$ at 25 and $50 \mathrm{~V}$, respectively. It is clear that electroosmosis would benefit EUF but long term experiments are needed to understand the practical limitation in large scale application.

\subsection{AHSs removal efficiency}

Fig. 8 shows AHSs removals in terms of UV absorbance at $254 \mathrm{~nm}$ at various experimental conditions. The unfractionated AHSs removals were $86 \%, 85 \%$ and $80 \%$ at 49,74 and $98 \mathrm{kPa}$, respectively and increased to more than $90 \%$ after applying voltages. It is clear that applying electric voltage could benefit AHSs removals. More AHSs were conveyed towards the membrane at higher pressure than those at lower pressure, and therefore the removal efficiency decreased as transmembrane pressure increased. The incremental AHSs rejections in the presence of electric field were contributed by electrophoresis which carries charged AHSs away

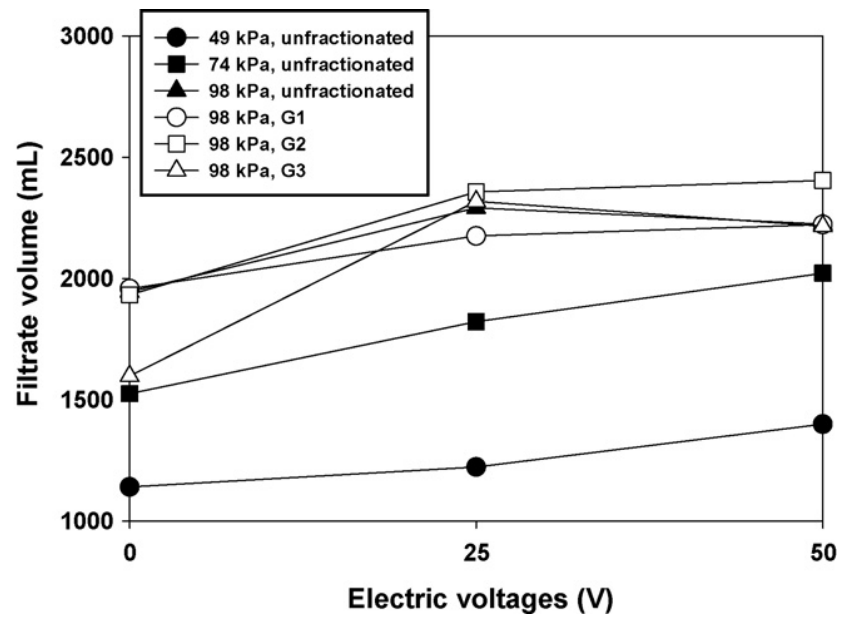

Fig. 7. Effect of voltage on water production.

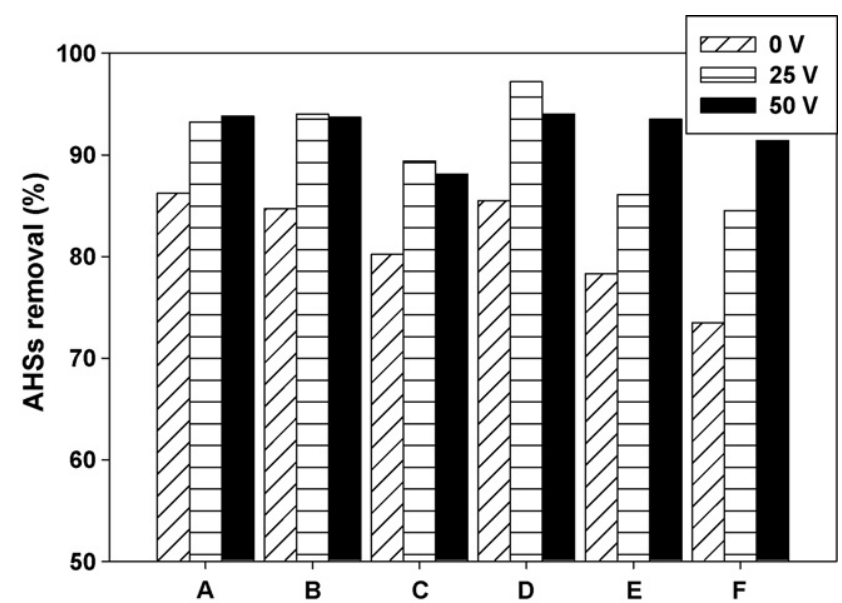

Fig. 8. AHSs rejections via EUF. A: AHSs, $49 \mathrm{kPa}$; B: AHSs, $74 \mathrm{kPa}$; C: AHSs, $98 \mathrm{kPa}$; D: G1 fraction, $98 \mathrm{kPa}$; E: G2 fraction, $98 \mathrm{kPa}$; and F: G3 fraction, $98 \mathrm{kPa}$.

from the membrane surface. The improvement of rejection was also observed during electrofiltration of proteins [23], oil wastewater [24] and silica wastewater [22]. The decrease in AHSs removal when electric voltage increased may be due to the electrochemical interactions between membrane and solution as described in Section 3.3.

Fig. 8 also demonstrates the MW effect on EUF performance. Without voltage, removal efficiency followed the MW order, i.e., $\mathrm{G} 1>\mathrm{G} 2>\mathrm{G} 3$. This is consistent with the result of Lin et al. [4]. After applying voltage, this trend remained. In general, more than $90 \%$ AHSs removal could be achieved at $50 \mathrm{~V}$. Furthermore, a decrease in AHSs removal for G1 group from 25 to $50 \mathrm{~V}$ indicated a change in characteristic of AHSs.

\section{Conclusions}

This study investigated the effect of pressure and AHSs MW on EUF. The AHSs were fractionated into G1, G2 and G3 groups which represented organics in 3000 to $>20,000,500$ to 3000 , and $<400$ to $500 \mathrm{Da}$, respectively. Without applying electrical voltages, flux declined in a descending order: $\mathrm{G} 3>\mathrm{G} 2>\mathrm{G} 1 \sim$ unfractionated. Based on flux, AHSs removal and AFM surface analysis results, a small MW group with low AEM would pass through membrane and cause severer fouling. After applying electrical voltages, both flux and AHSs removal were increased due to electrokinetic effects. However, the electrochemical reactions which took place in anode and cathode may influence membrane characteristics as well as AHSs composition. Therefore the results suggest that an inorganic membrane is more appropriate, if high electric voltage is applied during EUF.

\section{Acknowledgement}

This study was partly supported by a research grant, NSC922211-E-002-031, from National Science Council, Taiwan, ROC. The authors are thankful to Ms. Yeu-Harn Tsai for editing this article. The assistance of Dr. C.J. Chuang (R\&D Center for Membrane Technology, Chung Yuan Christian University) in providing streaming potential information is gratefully acknowledged.

\section{References}

[1] T. MacCormick, Cryptosporidium - driving the shift towards microfiltration? Filtr. Sep. 36 (1999) 16-19. 
[2] C.F. Lin, T.Y. Lin, O.J. Hao, Effects of humic substance characteristics on UF performance, Water Res. 34 (2000) 1097-1106.

[3] C.W. Li, Y.S. Chen, Fouling of UF membrane by humic substance: effects of molecular weight and powder-activated carbon (PAC) pre-treatment, Desalination 170 (2004) 59-67.

[4] C.F. Lin, Y.J. Huang, I.J. Hao, Ultrafiltration processes for removing humic substances: effect of molecular weight fractions and PAC treatment, Water Res. 33 (1999) 1252-1264.

[5] W. Yuan, A.L. Zydney, Humic acid fouling during ultrafiltration, Environ. Sci. Technol. 34 (2000) 5043-5050.

[6] T. Carroll, S. King, S.R. Gray, B.A. Bolto, N.A. Booker, The fouling of microfiltration membranes by NOM after coagulation treatment, Water Res. 34 (2000) 2861-2868.

[7] K.J. Howe, M.M. Clark, Fouling of microfiltration and ultrafiltration membranes by natural waters, Environ. Sci. Technol. 36 (2002) 3571-3576.

[8] W. Yuan, A.L. Zydney, Humic acid fouling during microfiltration, J. Membr. Sci. 157 (1999) 1-12.

[9] G. Akay, R.J. Wakeman, Electric field enhanced crossflow microfiltration of hydrophobically modified water soluble polymers, J. Membr. Sci. 131 (1997) 229-236.

[10] M. De Nobili, G. Bragato, A. Mori, Capillary electrophoretic behaviour of humic substances in physical gels, J. Chromatogr. A 863 (1999) 195-204.

[11] M. Hosse, K.J. Wilkinson, Determination of electrophoretic mobilities and hydrodynamic radii of three humic substances as a function of $\mathrm{pH}$ and ionic strength, Environ. Sci. Technol. 35 (2001) 4301-4306.

[12] Y.-H. Weng, K.-C. Li, Electrically enhanced crossflow ultrafiltration for treatment of water containing humic acid: a preliminary study, Adv. Asian Environ. Eng. 3 (2003) 60-66.

[13] Y.H. Weng, K.C. Li, L.H. Chaung-Hsieh, C.P. Huang, Removal of humic substances (HS) from water by electro-microfiltration (EMF), Water Res. 40 (2006) 1783-1794.
[14] Y.H. Weng, L.H. Chaung-Hsieh, H.H. Lee, K.C. Li, C.P. Huang, Removal of arsenic and humic substances (HSs) by electro-ultrafiltration (EUF), J. Hazard. Mater. 122 (2005) 171-176.

[15] F. Cooper, O. Mees, M. Bier, Water purification by forced-flow electrophoresis, J. Sanitary Eng. Div. Proc. Am. Soc. Civil Engineers 91 (1965) $13-25$.

[16] P. Hiemenz, R. Rajagopalan, Electrophoresis and other electrokinetic phenomena, in: Principles of Colloid and Surface Chemistry, third ed., Marcel Dekker, Inc., New York, 1997, 534-570.

[17] G.C.C. Yang, T.Y. Yang, S.H. Tsai, Crossflow electro-microfiltration of oxide-CMP wastewater, Water Res. 37 (2003) 785-792.

[18] Z. Lazarova, W. Serro, Electromembrane separation of mineral suspensions: influence of process parameters, Sep. Sci. Technol. 37 (2002) 515534.

[19] C. Chuang, C. Fang, K. Tung, Electro-microfiltration of colloidal suspensions, Sep. Sci. Technol. 38 (2003) 797-816.

[20] N.H. Lee, G. Amy, J.P. Croue, H. Buisson, Identification and understanding of fouling in low-pressure membrane (MF/UF) filtration by natural organic matter (NOM), Water Res. 38 (2004) 4511-4523.

[21] N. Lee, G. Amy, J.P. Croue, H. Buisson, Morphological analyses of natural organic matter (NOM) fouling of low-pressure membranes (MF/UF), J. Membr. Sci. 261 (2005) 7-16.

[22] G.C.C. Yang, C.J. Li, Electrofiltration of silica nanoparticle-containing wastewater using tubular ceramic membranes, Sep. Purif. Technol. 58 (2007) 159-165.

[23] R.J. Wakeman, Electrically enhanced microfiltration of albumin suspensions, Food Bioprod. Process. 76 (1998) 53-59.

[24] H.M. Huotari, I.H. Huisman, G. Tragardh, Electrically enhanced crossflow membrane filtration of oily waste water using the membrane as a cathode, J. Membr. Sci. 156 (1999) 49-60. 